REVIEW

\title{
Time trends in allergic disorders in the UK
}

\author{
R Gupta, A Sheikh, D P Strachan, H R Anderson
}

Thorax 2007;62:91-96. doi: 10.1136/thx.2004.038844

\begin{abstract}
See end of article for authors' affiliations

.....................

Correspondence to: Ms R Gupta, Division of Community Health Sciences, St George's, University of London, London SW17 ORE, UK; rgupta@sgul.ac.uk
\end{abstract}

Received 7 January 2006 Accepted 21 July 2006

\begin{abstract}
Background: Allergic disorders are common in the UK. This study reviews recent UK time trends in the prevalence, morbidity and mortality for allergic disorders, excluding asthma.

Methods: A trend analysis was performed over recent decades of national, representative or repeat surveys, primary care consultations, prescriptions, hospital admissions, and mortality.

Results: Serial surveys showed that the prevalence of diagnosed allergic rhinitis and eczema in children have both trebled over the last three decades. While these long term trends were paralleled by the prevalence of disease symptoms, more recent symptom prevalence data suggest a decline. Similarly, GP consultation rates rose by $260 \%$ for hay fever and by $150 \%$ for eczema overall during the period 1971-91, but rates have stabilised over the past decade. Hospital admissions for eczema have been stable since 1995, and hospital admissions for allergic rhinitis have fallen to about $40 \%$ of their 1990 levels. Since 1990, admissions for anaphylaxis have increased by $700 \%$, for food allergy by $500 \%$, for urticaria by $100 \%$, and for angiooedema by $40 \%$. Prescriptions issued for all types of allergy have increased since 1991.

Conclusions: The prevalence and healthcare usage for eczema and hay fever have increased substantially over recent decades, but may now be stabilising or even falling. In contrast, admissions for some systemic allergic diseases have risen sharply in the last decade which may indicate a rising incidence of these conditions. Although changes in treatment and other healthcare factors may have contributed to these trends, there may also be a change in the aetiology of allergic disease in the UK.
\end{abstract}

A llergic disorders are common and represent an important source of patient morbidity and healthcare usage. ${ }^{1}$ Epidemiological studies indicate that the prevalence of organ-specific allergic disorders such as allergic rhinitis, asthma, and eczema have increased in recent decades in many western countries..$^{1-5}$ Equally striking are geographical variations in the prevalence of these disorders in both children ${ }^{6}$ and adults. $^{7}$ Over the last decade other systemic allergic conditions including anaphylaxis and food allergy also appear to be increasing. ${ }^{8}$

This review brings together available information from major published surveys and national datasets about trends in the prevalence and healthcare usage of a range of conditions in which allergy is an important underlying mechanism. The conditions considered are allergic rhinoconjunctivitis, anaphylaxis, angio-oedema, atopic eczema, conjunctivitis, food allergy and urticaria, but asthma is excluded because it is the subject of a separate paper.

\section{METHODS}

Data sets

In order to maximise the generalisability of results, we focused on trend data from routine national health information sources and large national or regional surveys. These included the Health Survey for England (HSE) (1996-2001) $)^{2-5}$ and the International Study of Asthma and Allergies in Childhood (ISAAC) (1995-2002 $)^{49}$ for estimates of symptomatic and clinician diagnosed disease prevalence; Morbidity Statistics from General Practice (MSGP) (1971-91), ${ }^{10-13}$ the Royal College of General Practitioners Weekly Returns Service (RCGP WRS) (1990-2004), ${ }^{14}$ and Prescribing Cost Analysis (PCA) (1991$2004)^{15}$ data for primary healthcare usage; and Hospital Episode Statistics (HES) $(1990 / 1-2003 / 4)^{16}$ for data on hospital admissions. While the hospital admissions analysis for systemic allergic disorders (anaphylaxis, angio-oedema, food allergy and urticaria) presented here overlaps with previously published work, ${ }^{8}$ it has been extended to include additional diagnoses, analysis by age, and three additional years of data. Mid-year population estimates from the Office for National Statistics were used to calculate rates. ${ }^{17}$

\section{Definitions}

The working definitions used for our analyses were determined largely by the definitions used in the original data sets and studies. Where possible the definitions of allergic diseases were based on WHO International Classification of Diseases (ICD) codes for HES, RCGP WRS and MSGP4 data (table 1). The definitions from ISAAC and HSE are based on the questions used in those surveys. Children were defined as those aged $<15$ years; young adults as those aged 15-44 years; and older adults as those aged 45 years and over, except when the source of data restricted analysis to a different age range. Where trends are presented graphically, log scales are used to clarify the comparison of rates of change over time.

\section{RESULTS}

Prevalence

Trends in the 12 month period prevalence of symptoms of selfreported rhinoconjunctivitis are based on surveys at two time points. The ISAAC survey observed a decrease in 12-14 year olds from $18 \%$ in 1995 to $15 \%$ in 2002 (fig 1A). ${ }^{9}$ The HSE found no change in the 12 month period prevalence of symptoms ( $11 \%$ in children aged $2-15$ years and $15 \%$ in adults, unchanged between 1996 and 2001). ${ }^{24}$

Longer term trends are available for diagnosis, although the interpretation of these is complicated by possible changes in diagnostic practice. The prevalence of "hay fever ever" rose dramatically from $3.2 \%$ in 1964 to $12 \%$ in 1989 in Aberdeen schoolchildren ${ }^{18}$ and, in keeping with this, a comparison of two large cohorts showed that, in 16 year olds, the prevalence of hay fever doubled from $12 \%$ in 1974 to $23 \%$ in $1986 .{ }^{19}$ More recent serial health surveys suggest that the lifetime prevalence

Abbreviations: OSA, obstructive sleep apnoea; PSG, polysomnography 
Table 1 ICD codes for allergic conditions (used in admissions and GP data sets)

\begin{tabular}{lll}
\hline Condition & ICD-9 codes & ICD-10 codes \\
\hline Allergic rhinitis & 477 Allergic rhinitis & $\begin{array}{l}\text { J30.1 Allergic rhinitis due to } \\
\text { pollen } \\
\text { J30.2 Other seasonal } \\
\text { allergic rhinitis } \\
\text { J30.3 Other allergic rinitis } \\
\text { J30.4 allergic rhinitis, } \\
\text { unspecified }\end{array}$ \\
& & T78.0 Anaphylactic shock \\
due to adverse food reaction
\end{tabular}

of hay fever diagnosis continues to increase in children of all ages as well as in adults, but that the rate of increase may have declined since the latter part of the 1990s (fig 1B). ${ }^{249} 2021$

The results are very similar for symptoms and diagnosis of eczema. The 12 month prevalence of flexural rash in 12-14 year olds has also fallen during the last decade from 16\% in 1995 to $11 \%$ in $2002 .^{9}$ The 12 month prevalence of itchy skin ever has fallen slightly between 1996 and 2001 from $31 \%$ to $29 \%$ in children and from $38 \%$ to $36 \%$ in adults (fig 1A). ${ }^{2}{ }^{4}$ Earlier studies based on diagnosis showed that the prevalence of eczema ever in the Aberdeen children more than doubled from $5 \%$ in 1964 to $12 \%$ in $1989,,^{18}$ while the comparison of 16 year old cohorts showed that the prevalence of eczema doubled from $3 \%$ in 1974 to $6 \%$ in $1986 .{ }^{19}$ Again, recent serial surveys suggest that the lifetime prevalence of eczema has continued to increase in children of all ages, particularly in those aged under 5 years, but that the rate of increase may have slowed (fig 1B). ${ }^{4} 492021$

In repeat birth cohort surveys of 3-4 year olds in the Isle of Wight there was a threefold increase in peanut sensitisation from 1989 to $1994-6$ of $1.1 \%$ (95\% CI 0.6 to 2.0 ) to $3.3 \%$ (95\% CI 2.4 to 4.4 ) and a doubling-although statistically nonsignificant-in reported peanut allergy over the same period from $0.5 \%$ (95\% CI 0.2 to 1.1 ) to $1.0 \%$ (95\% CI 0.5 to 1.7 ). ${ }^{22}$

Among adults, national surveys have found that there has been no recent increase in the population geometric mean total IgE (45 in men and 30 in women in 1995-6 and 42 in men and 27 in women in 2001). ${ }^{24}$

\section{General practice consultations}

Decennial national surveys of about 70 general practices in England and Wales showed that, between 1971 and 1991, the proportions of patients consulting GPs more than doubled in all age groups for allergic rhinitis (ICD9 477), increasing from 1100 to 2830 per 100000 patient years overall (table 2). ${ }^{10-13}$ The rate of increase appeared highest in children and the oldest adults, with nearly a trebling in both these age groups compared with an increase of 2.3 times in 15-44 year olds. Patients consulting for eczema (ICD9 691 and 692) also increased from 3430 to 5060 per 100000 patient years over the same period. Rates doubled in children to 9705 per 100000 in 1991 with more modest increases in adults.

Proportions of patients consulting for urticaria (ICD9 708) were recorded as falling slightly over these three decades overall. This was true for children and young adults (aged 1544 years) while, in older adults, the rates rose from 496 to 610 per 100000 person years.

Weekly primary care consultation rates (which count episodes rather than patients), collected for a national sample of GP practices over the last decade, show varying trends (fig 2). ${ }^{14}$ Consultations for allergic rhinitis (ICD9 477) were stable from 1981 to $1990^{23}$ but rose to a transitory peak of 44 per 100000 per week in 1992 (which may have affected the rates in the GP survey carried out in 1991/92). Since then, consultation rates have fallen steadily in school age children and younger adults but appear to have stabilised in other age groups with an overall weekly episode rate of 20 per 100000 in 2004.

Weekly consultation rates for atopic dermatitis and related conditions (ICD9 691) have fallen moderately during the latter part of the last decade from 25 per 100000 in 1995 to 20 in 2002 , but stood at 22 in 2004. There are, however, differing agespecific trends, with falls occurring in pre-school children (240 $v 168$ per 100000 in $0-4$ year olds) but rises occurring in older adults ( $6 v 10$ per 100000 in 45-64 year olds) and a doubling in those aged over 65 years $(6 v 14$ per 100000$)$.

\section{Primary care prescribing}

Available data on community prescriptions are shown in fig 3. The numbers of community prescriptions for drugs used for nasal allergy (British National Formulary 12.2.2) and anaphylaxis (BNF 3.4.3) have risen steadily in patients of all ages over the last 20 years; a similar trend is also evident for antiinflammatory eye preparations (BNF 11.4.2) which are commonly used for ocular allergy. From 1991 to 2004, prescriptions for all nasal allergy increased by over $60 \%$ (fig 3 ) from 2.7 million to 4.5 million, those for anaphylaxis increased 12-fold to 124000 , and ocular anti-inflammatory prescriptions increased by $50 \%$ to 1.4 million. Prescriptions for skin emollients have doubled in the decade to 11.5 million, while those for topical corticosteroids have remained stable at 12.2 million. These skin preparations may, however, have been prescribed for dermatological conditions other than eczema. We do not know to what extent trends are explained by trends in numbers of persons prescribed these drugs or by an increase in prescriptions per affected person.

\section{Hospital admissions}

Trends in hospital admissions for the time period 1990/1 to 2003/4 show continuing increases in admission rates for a range of systemic allergic conditions including anaphylaxis, angiooedema, food allergy, and urticaria (figs 4A-D). ${ }^{8}$ These admission data do not include periods in accident and emergency departments for observation and are therefore likely to underestimate the actual incidence or prevalence of these conditions. Admission rates for anaphylaxis, although relatively low, have risen sevenfold from 5 per million of population in 1990/1 to 36 in 2003/4, and this has been reflected in all age groups. Rates for angio-oedema were stable in children and younger adults over this period but doubled in those aged over 45 years from 11 to 23 per million. Food allergy admissions rose from 5 to 26 per million; this was particularly apparent in children where rates were highest and rose nearly sevenfold from 16 to 107 per million. Admissions for urticaria more than 

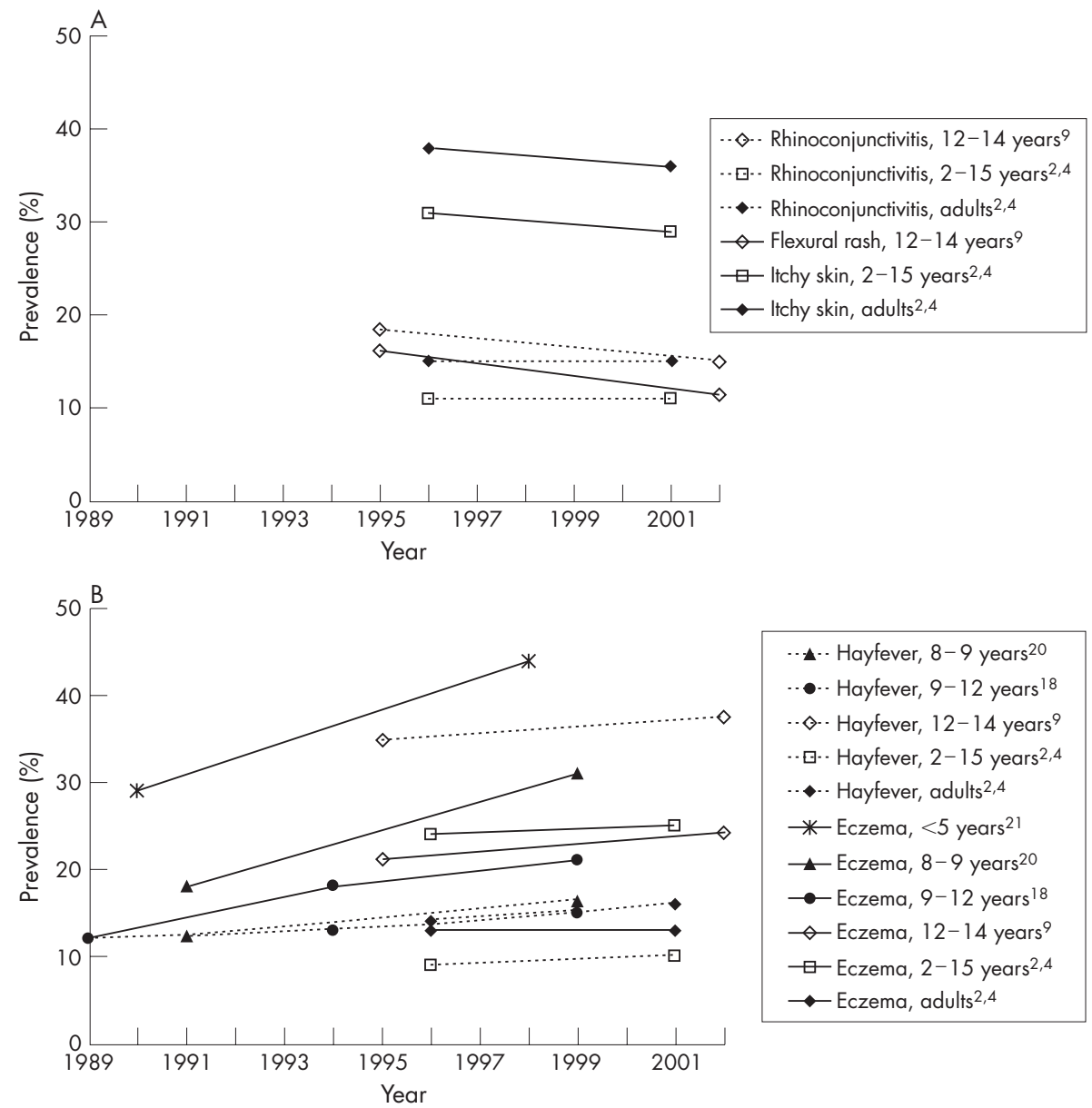

Figure 1 (A) Trends in prevalence of symptoms of hay fever and eczema in children and adults, 1989-2002. (B) Trends in prevalence of hay fever and eczema diagnoses ever in children and adults, 1989-2002.

doubled from 20 to 44 per million and, again, both admission rates and rates of increase were greatest in children.

Trends for admissions for other allergic disorders are less clear and more difficult to interpret (fig $4 \mathrm{E}-\mathrm{G}$ ). Trends for atopic dermatitis and conjunctivitis are shorter due to a change in the admissions coding definitions in 1995/6. Since then, rates have been stable in all age groups for atopic dermatitis and stood at 32 per million in 2003/4 overall. The very small number

Table 2 Patients consulting GP for hay fever, eczema and urticaria in England and Wales, 1971-91

\begin{tabular}{lrrr}
\hline & 1971 & 1981 & 1991 \\
\hline Allergic rhinitis/hay fever & & & \\
All ages & 1100 & 1970 & 2830 \\
$0-14$ & 1354 & 2754 & 4006 \\
$15-44$ & 1572 & 2539 & 3639 \\
$45+$ & 420 & 730 & 1250 \\
Eczema & 1971 & 1981 & 1991 \\
All ages & 3430 & 3410 & 5060 \\
$0-14$ & 4760 & 4963 & 9705 \\
$15-44$ & 3076 & 3135 & 4142 \\
$45+$ & 2943 & 2840 & 4080 \\
Urticaria & 1971 & 1981 & 1991 \\
All ages & 840 & 830 & 710 \\
$0-14$ & 1390 & 1260 & 1109 \\
$15-44$ & 826 & 890 & 668 \\
$45+$ & 496 & 520 & 610 \\
\hline Data shown as rates per 100000 person years at risk.
\end{tabular}

of admissions recorded as conjunctivitis in the years after the coding change makes trends hard to interpret in adults but, in children, they appear to be stable at 4 per million. Admissions for allergic rhinitis have fallen from 35 to 14 per million since 1990 and the decrease has occurred in all age groups.

\section{Mortality}

A study of hospital admissions and deaths from anaphylaxis showed there were 12 deaths from 1991 to $1995 .{ }^{24}$ This is likely to be an underestimate because some deaths from allergic conditions may be recorded as being caused by asthma.

\section{DISCUSSION}

This review has collated information on trends in allergic diseases (excluding asthma) in the UK from the earliest available data to the present day. We used only data sources from national routine data or large scale surveys and cohort studies designed to properly investigate time trends in prevalence and healthcare usage. There are few sources which meet these criteria, and they cover only some of today's common allergic diseases.

The prevalence studies of eczema and hay fever use measures of self-reported symptoms (often over a set period such as l year due to the transient nature of some symptoms) as well as using these labels specifically or sometimes as self-reports of doctor diagnosis. There is no way to validate these recalled symptoms or the diagnoses given, and it is known that the pattern of diagnosing can change over time independently of 


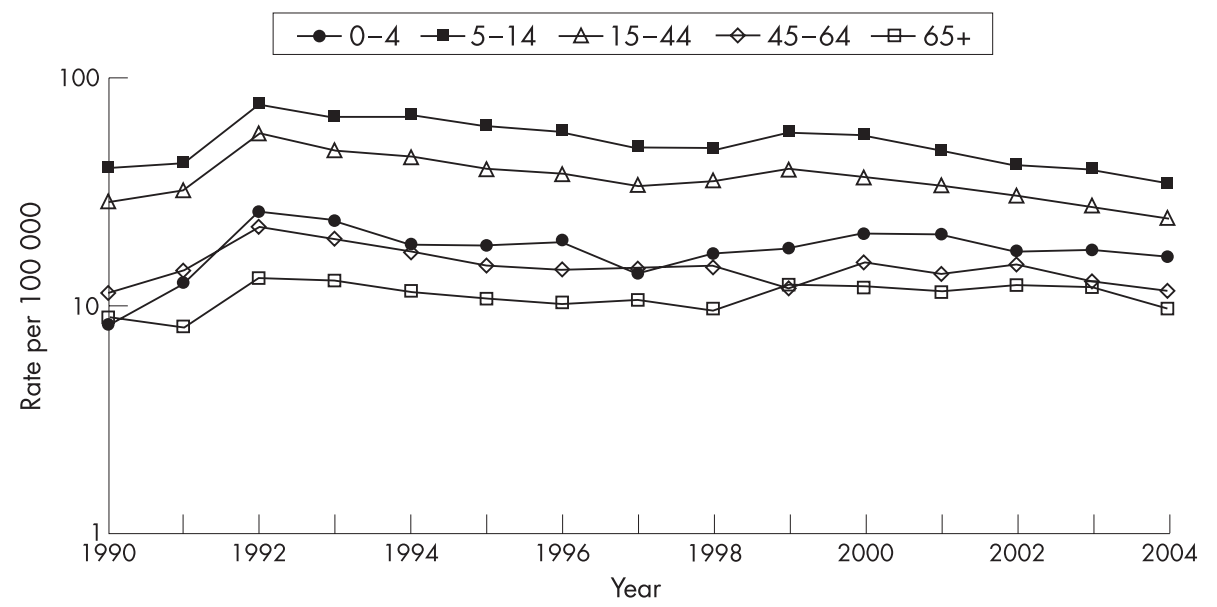

Figure 2 Trend in weekly GP episodes for hay fever, England \& Wales 1990-2004.

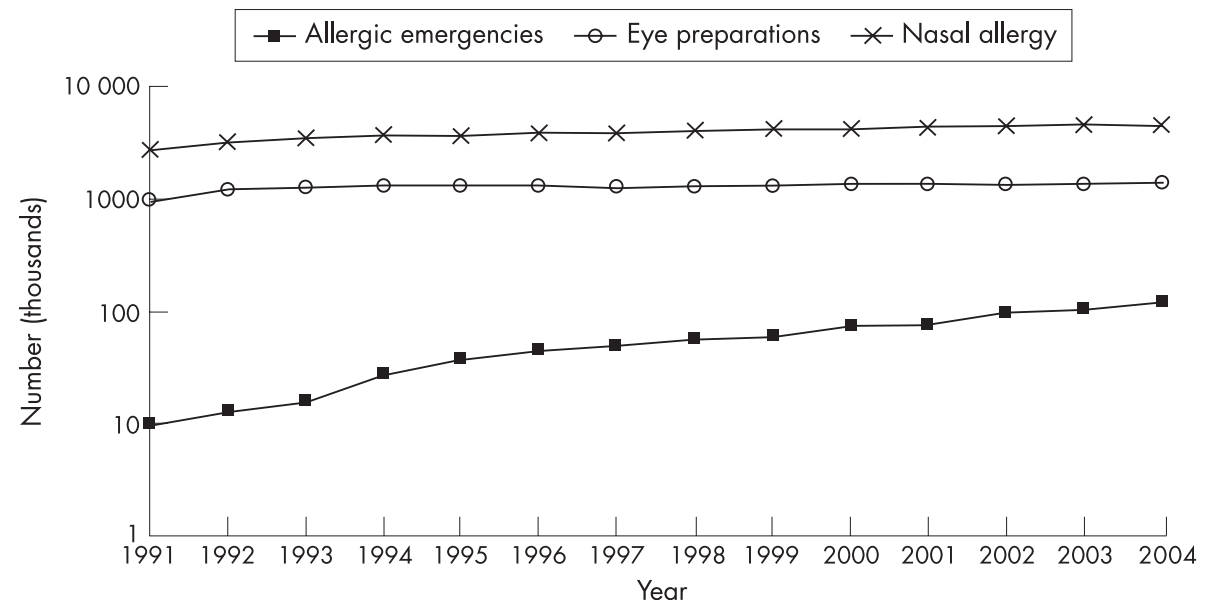

Figure 3 Trends in the number of prescriptions for allergic emergencies, anti-inflammatory eye preparations and nasal allergy, England 1991-2004. A logarithmic $y$ axis is used to facilitate interpretation of trends in data that show large variations in scale.

symptoms experienced. ${ }^{25}$ Trends in healthcare usage may reflect changes in the labelling or coding of diseases, changes in healthcare patterns (by the behaviour of patients or doctors, changes in treatment or changes in the health system), as well as changes in the underlying epidemiology of the condition. The decennial surveys of GP consultations and the current Royal College of General Practitioners weekly collation of episodes use a national and broadly representative sample of general practices in England and Wales, although inner cities are under-represented. They report on a specific set of diagnoses so that, in some cases (for example, with urticaria from the decennial surveys), it is impossible to comment on possible diagnostic transfer to or from other conditions such as angio-oedema. Both GP visits and hospital admissions may be affected by changes in thresholds for the use of these services, but we have no evidence on this for the conditions considered or on trends in visits to accident and emergency or outpatient departments. It must also be remembered that both these sources report on episodes not patients so, for example, trends in admissions can be affected by the pattern of readmissions. However, we consider these sources to offer the best available evidence on the trends in allergic disease in the UK.

The prevalence of a diagnosis of eczema and hay fever in children has more than trebled since the 1960s. Since the mid 1990s there is evidence that, while the prevalence of a diagnosis (ever) has stabilised or increased a little, there has been a stabilisation or fall in the 12 month period prevalence of the symptoms of these disorders. We note, however, that a cohort of UK adults showed a small increase in the prevalence of nasal allergy over the last decade from $29 \%$ to $32 \%$ (S Chinn, personal communication). ${ }^{26}$ There is also some evidence of a rise in the prevalence of peanut allergy in children. GP consultations for eczema and hay fever show a similar trend with rates doubling overall since 1971, but with smaller rises or falls in all age groups since the mid 1990s. In contrast, consultations recorded as urticaria have fallen overall since 1970. Community prescribing for nasal and ocular allergies have risen since 1980, as have prescriptions for dry skin conditions. The number of prescriptions for anaphylaxis has increased more than tenfold since 1991. Admissions for atopic dermatitis and conjunctivitis are harder to interpret over the last decade but appear to be stable while those for allergic rhinitis have fallen. Admissions for the systemic allergic conditions anaphylaxis, angio-oedema, food allergy and urticaria, though rare, have increased since 1990 with particularly dramatic rises for anaphylaxis and food allergies.

Taken together, these patterns of prevalence, prescribing, and healthcare use suggest a rise in eczema and hay fever in recent decades with a stabilisation more recently, not entirely due to changes in health care or treatment of these conditions. The 
$-0-14-15-44 \triangle 45+$
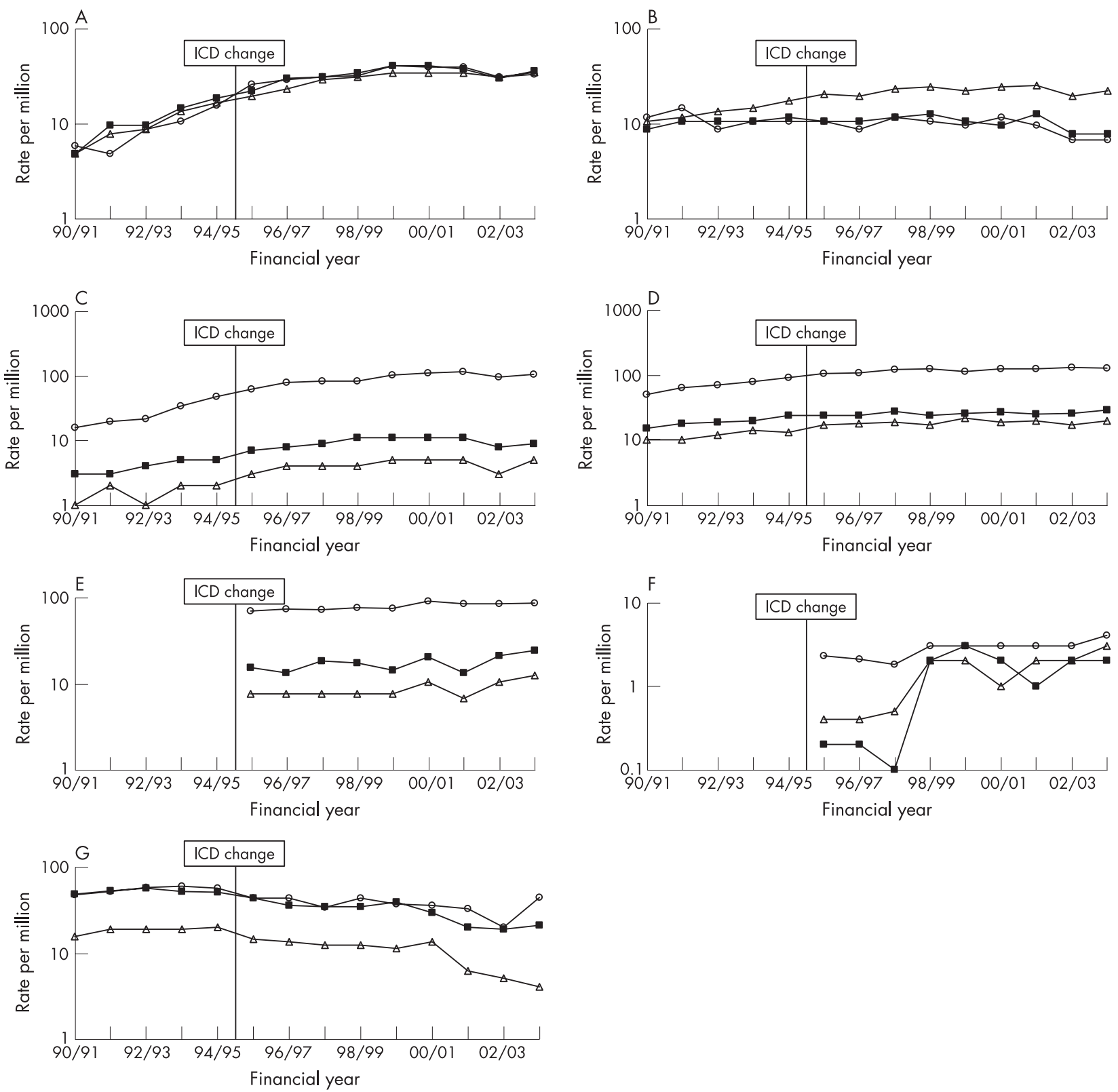

Figure 4 Trends in hospital admission rates for anaphylaxis by age in England during the period 1990-2004 for (A) anaphylaxis, (B) angio-oedema, (C) food allergy, (D) urticaria, $(E)$ atopic dermatitis, $(F)$ conjunctivitis, and $(G)$ allergic rhinitis.

pattern for anaphylaxis looks very different with large increases recently in both prescribing and admissions. Admissions for food allergy also seem to have risen substantially with some evidence of a rise in prevalence in children. Admissions for urticaria and angio-oedema-for which diagnoses may overlap with food allergies-have risen sharply in contrast to reported falls in GP consultations for urticaria over the last decades.

UK data on the epidemiology and disease burden associated with allergic disorders are among the best in the world. However, these conclusions are based on very limited information, hence the need for ongoing surveillance. Some of the trends could be explained by artefacts such as changes in perceptions and diagnostic practices over time. Other components of these trends may reflect changes in medical care such as improved treatment. Finally, these trends may reflect changes in the epidemiology resulting from changes in the sensitivity of the population or to changes in environmental factors that initiate or aggravate these conditions. ${ }^{27-29}$ The data themselves give few clues to the relative importance of these various factors. However, they are valuable for generating hypotheses, encouraging the development of better information systems for recording trends, and for anticipating the burden of allergic disease on the population and healthcare system.

\section{ACKNOWLEDGEMENTS}

The authors thank the Lung and Asthma Information Agency (LAIA) for allowing access to data. LAIA is funded by Asthma UK, the British Thoracic Society and the British Lung Foundation. They also thank the British Society for Allergy and Clinical Immunology for their contribution to the funding of this work. 


\section{Authors' affiliations}

R Gupta, Lung and Asthma Information Agency, Division of Community Health Sciences, St George's, University of London, UK

A Sheikh, Division of Community Health Sciences: GP Section, University of Edinburgh, UK

D P Strachan, H R Anderson, Division of Community Health Sciences, St George's, University of London, UK

Competing interests: None.

\section{REFERENCES}

1 Gupta R, Sheikh A, Strachan DP, et al. Burden of allergic disease in the UK: secondary analyses of national databases. Clin Exp Allergy 2004;34:520-6.

2 Prescott-Clarke P, Primatesta P, eds. The Health Survey for England 1996. London: The Stationery Office, 1998.

3 Prescott-Clarke P, Primatesta P, eds. The Health Survey for England, 1997: The health of young people 1995-1997. London: The Stationery Office, 1999.

4 Bajekal M, Primatesta P, Prior G, eds. Health Survey for England 2001 : Respiratory symptoms, atopic conditions and lung function. London: The Stationery Office, 2003.

5 Sproston K, Primatesta P, eds. The Health Survey for England 2002: The health of children and young people. London: The Stationery Office, 2004

6 International Study of Asthma and Allergies in Childhood. Worldwide variation in prevalence of symptoms of asthma, allergic rhinoconjunctivitis, and atopic eczema: ISAAC. Lancet 1998;351:1225-32.

7 European Community Respiratory Health Survey. Variations in the prevalence of respiratory symptoms, self-reported asthma attacks, and use of asthma medication in the European Community Respiratory Health Survey (ECRHS). Eur Respir J 1996:9:687-95.

8 Gupta R, Sheikh A, Strachan DP, et al. Increasing hospital admissions for systemic allergic disorders in England: analysis of national admissions data. BMJ 2003;327:1142-3.

9 Anderson HR, Ruggles R, Strachan DP, et al. Trends in prevalence of symptoms of asthma, hay fever, and eczema in 12-14 year olds in the British Isles, 19952002: questionnaire survey. BMJ 2004;328:1052-3.

10 Office for National Statistics. Morbidity statistics from general practice 198182 , Series MB5 No 1 .

11 Office for National Statistics. Morbidity statistics from general practice 197071. Studies on Medical and Population Subjects, No. 26.
12 Office for National Statistics. Morbidity statistics from general practice 199192, Series MB5 № 3.

13 Office for National Statistics. Morbidity statistics from general practice 199192: Patient records.

14 Weekly Returns Service. Annual report 2004. Birmingham: Royal College of General Practitioners, 2004. http://www.rcgp.org.uk/default.aspx?page $=440$ (accessed April 2006).

15 Department of Health. Prescription cost analysis. http://www.dh.gov.uk/ PublicationsAndStatistics/Publications/PublicationsStatistics/fs/en laccessed April 2006).

16 Department of Health. Hospital episode statistics. http://www.hesonline.nhs.uk (accessed April 2006).

17 Office for National Statistics. Mid-year population estimates. http:// www.statistics.gov.uk (accessed October 2004).

18 Devenny A, Wassall H, Ninan T, et al. Respiratory symptoms and atopy in children in Aberdeen: questionnaire studies of a defined school population repeated over 35 years. BMJ 2004;329:489-90.

19 Butland B, Strachan D P, Lewis S, et al. Investigation into the increase in hay fever and eczema at age 16 observed between 1958 and 1970 British birth cohorts. BMJ 1997;315:717-24.

20 Kwong G, Proctor A, Billings $C$, et al. Increasing prevalence of asthma diagnosis and symptoms in children is confined to mild symptoms. Thorax 2001;56:312-4

21 Kuehni CE, Davis A, Brooke AM, et al. Are all pre-school wheezing disorders increasing in prevalence? Lancet 2001:357:1821-5.

22 Grundy J, Matthews S, Bateman B, et al. Peanut allergy in three year old children - a population based study. J Allergy Clin Immunol 2001;107(Suppl):S231.

23 Ross AM, Fleming DM. Incidence of allergic rhinitis in general practice, 198192. BMJ 1994;308:897-900.

24 Sheikh A, Alves B. Hospital admissions for acute anaphylaxis: time trend study. BMJ 2000;320:1441.

25 Anderson HR, Butland BK, Strachan DP. Trends in prevalence and severity of childhood asthma. BMJ 1994:308:1600-4.

26 Chinn S, Jarvis D, Burney P, et al. Increase in diagnosed asthma but not in symptoms in the European Community Respiratory Health Survey. Thorax 2004; $59: 646-51$

27 Custovic A, Woodcock A. Exposure and sensitization in infants and children Curr Opin Allergy Clin Immunol 2001;1:125-6.

28 Sheikh A, Strachan DP. The hygiene theory: fact or fiction? Curr Opin Otolaryngol Head Neck Surg 2004;12:232-6.

29 Law M, Morris JK, Wald N, et al. Changes in atopy over a quarter of a century, based on cross sectional data at three time periods. BMJ 2005;330:1187-8. 